\title{
ALDR Enhanced Endothelial Injury in Hyperuricemia Screened using SILAC
}

\author{
Yang Zhang ${ }^{\mathrm{a}, \mathrm{d}}$ Quan Hong ${ }^{\mathrm{a}, \mathrm{d}}$ Zhiyong Huanga,b Peng Xue ${ }^{\mathrm{c}}$ Yang Lv ${ }^{\mathrm{a}}$ Bo Fu \\ Xiangmei Chen ${ }^{\mathrm{a}} \mathrm{Di} \mathrm{Wu}^{\mathrm{a}}$
}

aState Key Laboratory of Kidney Disease (2011DAV00088), Kidney Department \& Institute of Nephrology, Division of Clinical Internal Medicine, Chinese People's Liberation Army General Hospital, Beijing, bDepartment of Nephrology, The 175th Hospital of PLA, Zhangzhou, Fujian, 'Laboratory of Proteomics, Institute of Biophysics, Chinese Academy of Sciences, Beijing, P.R. China; ${ }^{d}$ These authors contributed equally to this work

\section{Key Words}

Uric Acid • Endothelial Injury • ALDR • Oxidative Stress • SILAC

\begin{abstract}
Background: The exact etiology of hyperuricemia-induced endothelial injury remains illdefined. To elucidate the mechanism that leads to endothelial injury in hyperuricemia, we investigated proteins expressed in human umbilical vein endothelial cells (HUVECs) cultured with high concentrations of uric acid (HUA) in vitro. Methods: We used stable isotope labeling with amino acids in cell culture (SILAC) combined with LC-MS/MS analysis to compare proteins expressed in HUVECs cultured in media with or without HUA. The results were confirmed by Western blotting. Reactive oxygen species (ROS) were detected using a confocal microscope. Results: Thirty-nine proteins with various cellular functions were differentially expressed. Among them, aldose reductase (ALDR) protein expression was enhanced significantly, indicating increased aldehyde reductase and oxidoreductase activities. ROS levels decreased when ALDR protein activity was inhibited by siALDR. Conclusions: ALDR protein may play an important role in endothelial injury induced by hyperuricemia, and activity of the ALDR protein is associated with oxidative stress.
\end{abstract}




\section{Cellular Physiology and Biochemistry}

Cell Physiol Biochem 2014;33:479-490

DOI: $10.1159 / 000358628$

Published onlıne: February 14, 2014

\section{Introduction}

Uric acid (UA) is the end product of purine nucleotide metabolism in humans [1-3]. Hyperuricemia results from either increased generation or decreased degradation of UA, which is biologically active and can lead to endothelial injury, oxidative stress, inflammation and vasoconstriction [4]. Hyperuricemia is an independent risk factor for many diseases, such as kidney and cardiovascular diseases [5]. Hyperuricemia contributes to endothelial dysfunction in early and middle stage chronic kidney disease (CKD), and oxidative stress is associated with this injury [5]. Previous studies have confirmed multiple mechanisms involved in endothelial dysfunction, including reduced nitric oxide (NO) bioavailability due to inhibited uptake of L-arginine, promotion of L-arginine degradation, and NO scavenging [4]. Park and colleagues suggested that high concentrations of uric acid (HUA) attenuated binding between endothelial nitric oxide synthase (eNOS) and calmodulin $(\mathrm{CaM})$, consequently decreasing eNOS activity and NO production [6]. They also reported that endothelial dysfunction is related to mitochondrial changes and decreased intracellular adenosine triphosphate (ATP) [7]. Another study indicated that HUA can induce endothelial dysfunction, and this effect is associated with oxidative stress [8]. However, further studies are needed to elucidate the comprehensive mechanism leading to endothelial injury induced by HUA. Therefore, we used a new method, stable isotope labeling by amino acids in cell culture (SILAC), combined with liquid chromatograph-mass spectrometry (LC-MS), to analyze the differentially expressed proteins in human umbilical vein endothelial cells (HUVECs) cultured with HUA compared with those without UA. This new LC-MS analysis improves upon the shortcomings of previous MS analysis, and SILAC (first reported by Mann et al.) can be used to analyze differentially expressed proteins both qualitatively and quantitatively, which greatly improves the sensitivity and accuracy of measurements [9]. Gene Ontology (GO) classification and the Molecular Annotation System (MAS) were used to confirm the characteristics of these differentially expressed proteins and the interactions among them.

Here, we used the HUVEC line to screen differentially expressed proteins in cells exposed to HUA using SILAC combined with LC-MS analysis (http://www.uniprot.org and http://bioinfo.capitalbio.com/mas3/ online analysis platforms).

\section{Materials and Methods}

\section{Main reagents}

SILAC $^{\text {TM }}$ kits and RNAi MAX transfection reagent were purchased from Invitrogen (Carlsbad, CA, USA). Trypsin and uric acid were purchased from Promega (Madison, USA). Antibodies were obtained from Santa Cruz Biotechnology (California, USA). Oxonic acid potassium salt was bought from Sigma (ST. LOUIS, USA), and the Nano-ESI LTQ-ion trap mass spectrometer was purchased from Thermo Fisher Scientific (Waltham, USA)

\section{Cell culture}

The human umbilical vein endothelial cell line (HUVEC) was purchased from the American Type Culture Collection (ATCC, Manassas, VA) and was cultured in RPMI 1640 media (Gibco Laboratories, Grand Island, NY) containing $10 \%$ fetal bovine serum (FBS), $100 \mathrm{U} / \mathrm{L}$ penicillin, and $100 \mathrm{U} / \mathrm{L}$ streptomycin at $37^{\circ} \mathrm{C}$ under $5 \% \mathrm{CO}_{2}$. Two days prior to experiments, HUVECs were subcultured in $35 \mathrm{~mm}$ confocal glass bottom dishes or $25 \mathrm{~cm}^{2}$ bottles, as needed.

\section{Stable heavy isotope labeling}

HUVECs were cultured in RPMI 1640 media containing $0.1 \mathrm{mg} / \mathrm{mL}$ heavy-Lys $\left({ }^{13} \mathrm{C}_{6}\right.$-Lys) or $0.1 \mathrm{mg} /$ $\mathrm{mL}$ light-Lys for six generations to ensure isotope labeling efficiency, according to the manufacturer's instructions (Invitrogen). The media were replenished with fresh RPMI 1640 containing $0.1 \mathrm{mg} / \mathrm{mL}$ heavyLys $\left({ }^{13} \mathrm{C}_{6}\right.$-Lys) or $0.1 \mathrm{mg} / \mathrm{mL}$ light-Lys during cell passaging, and the labeling efficiency reached $99 \%$ after 


\section{Cellular Physiology and Biochemistry}

Cell Physiol Biochem 2014;33:479-490

\begin{tabular}{l|l}
\hline DOI: $10.1159 / 000358628$ & (C) 2014 S. Karger AG, Basel
\end{tabular}

www.karger.com/cpb

Zhang et al.: ALDR Enhanced ECs Injury in Hyperuricemia
481

six generations. Cells were seeded in $25 \mathrm{~cm}^{2}$ culture flasks and classified into two groups: cells cultured in light-Lys media (non-UA) and those cultured in heavy-Lys containing HUA. Uric acid (600 $\mu$ M; Sigma) was added to the media for $24 \mathrm{~h}$. Cells were scraped, centrifuged, counted, and mixed at a 1:1 ratio for protein extraction.

\section{LC-MS analysis and data processing}

Extracted proteins were assessed by two-dimensional LC-MS as reported previously [9]. Data from MS analysis were searched against the NCBI Human refseq database (v.2011) using SEQUEST v.28 of Bioworks 3.31. The false discovery rate was calculated in the reverse database using trypsin as a search parameter and including up to two missed cleavage sites [9]. Initial mass deviations of precursor and fragment ions were up to $2 \mathrm{Da}$ and $1 \mathrm{Da}$, respectively. Mass deviations of amino acid modifications were set to $57.02 \mathrm{Da}$ and 19.02 Da for alkylated cysteine and oxidated methionine, respectively. Peptide possibility was calculated by Bioworks. The false discovery rate reached $1 \%$ after Xcorr, sp, Rsp, DeltaCn, and peptide possibility filtration. Protein coverage rate was calculated by a protein coverage summarizer. Pathway networks and protein functional grouping were generated by the online platform at http://bioinfo.capitalbio.com/mas3.0/.

\section{Small interfering RNA (siRNA) transfection}

ALDR siRNA (siALDR) (100 nM, sc-29204, Santa Cruz) was transfected into HUVECs using RNAi MAX transfection reagent according to the manufacturer's instructions (Invitrogen). A non-silencing siRNA was used as a negative control. Forty-eight hours after transfection, cells were starved for $16 \mathrm{~h}$, followed by treatment with UA, or no treatment.

\section{Western blot analysis}

RIPA lysis buffer $(50 \mathrm{mmol} / \mathrm{L}$ Tris- $\mathrm{HCl}, \mathrm{pH} 7.5,150 \mathrm{mmol} / \mathrm{L} \mathrm{NaCl}, 0.5 \%$ deoxycholate, $1 \%$ Nonidet P-40, $0.1 \%$ sodium dodecyl sulfate [SDS], $1 \mathrm{mM}$ PMSF, and a variety of protease inhibition agents [1 $\mu \mathrm{g} /$ $\mathrm{mL}]$ ) was used to extract protein. Cell lysates were used to determine protein concentration using a BCA kit. Approximately $80 \mu \mathrm{g}$ protein was loaded onto $10 \%$ SDS-PAGE, transferred to a polyvinylidene fluoride (PVDF) membrane, and kept overnight in $5 \%$ non-fat milk at $4^{\circ} \mathrm{C}$ after Ponceau S staining. The membrane was incubated in primary antibodies (Santa Cruz). Blots were developed with enhanced chemiluminescent (ECL) reagent (Santa Cruz) according to the manufacturer's instructions and exposed to X-ray film. Protein bands were quantified using quantity one software (Bio-Rad).

\section{Establishment of hyperuricemic mice models}

Hyperuricemic mice were established as described previously with slight modifications [10]. The animal protocol was reviewed and approved by the Institutional Animal Care and Use Committee of the Chinese PLA General Hospital. Wild-type C57BL/6 mice obtained from the Experimental Animal Center of Academy of Military Medical Sciences (China) were used as controls. Mice were housed in temperaturecontrolled cages on a 12-h light-dark cycle and given free access to water and normal chow diet. After 1 week of breeding for adaptation, mice were grouped into control $(n=6)$ or hyperuricemic $(n=6)$ groups. Mice were injected intraperitoneally with $250 \mathrm{mg} / \mathrm{Kg} \cdot \mathrm{d}$ oxonic acid potassium salt (Sigma) and $250 \mathrm{mg} /$ $\mathrm{Kg} \cdot \mathrm{d}$ uric acid (Sigma). Fourteen days after modeling, UA levels in the blood were evaluated, and aortas were subjected to immunohistochemistry.

\section{Immunohistochemistry}

Aortas were fixed in formalin and embedded in paraffin. Paraffin sections were cut into sections (3-4 $\mu \mathrm{m}$ thick) and mounted onto poly-L-lysine-coated slides. Endogenous peroxidase was blocked with $3 \%$ hydrogen peroxide. The sections were heated in a microwave oven for $10 \mathrm{~min}$ in sodium citrate buffer $\mathrm{pH}$ 6.0) incubated for 20 min with $1.5 \%$ normal goat serum, followed by overnight incubation with the primary antibody (AR; dilution 1:100). As a negative control, sections were incubated with phosphate-buffered saline (PBS). After removal of unbound primary antibody, the sections were incubated for $60 \mathrm{~min}$ at room temperature with biotinylated secondary antibody. Sections were rinsed and incubated for $60 \mathrm{~min}$ with avidin-biotinylated horseradish peroxidase (Vectastain Elite ABC Kit; Vector Laboratories). Incubation with 3,3-diaminobenzidine tetrahydrochloride as a substrate chromogen solution to produce a brown color was performed for $10 \mathrm{~min}$. Finally, the sections were counterstained with hematoxylin. 


\section{Cellular Physiology $\quad$ Cell Physiol Biochem 2014;33:479-490

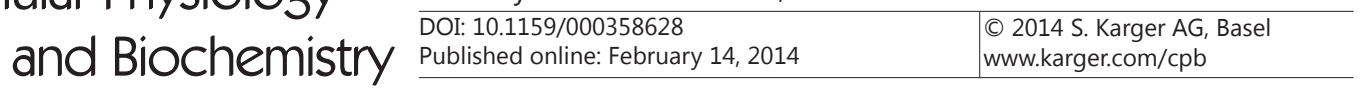 Zhang et al.: ALDR Enhanced ECs Injury in Hyperuricemia}

Table 1. Different proteins expression in endothelial cells cultured with high UA and without UA. * ALDR expression increased in endothelial cells cultured at the presence of $600 \mu \mathrm{mol} / \mathrm{L} \mathrm{UA}$ for $24 \mathrm{~h}$

\begin{tabular}{|c|c|c|c|c|}
\hline Uniprot KB & Protein Name & Definition & Mass & NRatio \\
\hline P15121 & ALDR & aldo-keto reductase family 1 , member $\mathrm{B} 1$, aldose reductase & 35830 & 18.97 \\
\hline B2RA03 & B2RA03 & keratin, type II cytoskeletal 18 & 48029 & 1.74196 \\
\hline Q53HU8 & Q53HU8 & vimentin & 53619 & 0.98813 \\
\hline Q9UII2 & ATIF1 & putative uncharacterized protein dkfzp564g0422 & 12397 & 0.98152 \\
\hline Q7L4M3 & Q7L4M3 & keratin, type II cytoskeletal 8 & 53671 & 0.93764 \\
\hline Q6IPT9 & Q6IPT9 & elongation factor 1 -alpha 1 & 50109 & 0.90875 \\
\hline P08729 & $\mathrm{K} 2 \mathrm{C} 7$ & keratin, type II cytoskeletal 7 & 51373 & 0.8926 \\
\hline Q6NUR7 & Q6NUR7 & ezrin & 69370 & 0.87473 \\
\hline B4DTN7 & B4DTN7 & isoform 1 of protein-glutamine gamma-glutamyltransferase 2 & 77280 & 0.7267 \\
\hline P26599 & PTBP1 & isoform 1 of polypyrimidine tract-binding protein 1 & 57186 & 0.72021 \\
\hline Q9H6X9 & Q9H6X9 & heat shock protein hsp 90 -beta & 83212 & 0.71646 \\
\hline Q86SX1 & Q86SX1 & isoform 2 of heat shock protein hsp 90 -alpha & 98099 & 0.6924 \\
\hline B4DTG2 & B4DTG2 & highly similar to elongation factor 1-gamma & 56114 & 0.68625 \\
\hline B7Z597 & B7Z597 & $60 \mathrm{kDa}$ heat shock protein, mitochondrial & 61016 & 0.6479 \\
\hline Q00610 & CLH1 & isoform 1 of clathrin heavy chain 1 & 191493 & 0.61413 \\
\hline P68366 & TBA4A & tubulin alpha-4a chain & 49892 & 0.577 \\
\hline P22626 & ROA2 & isoform $b 1$ of heterogeneous nuclear ribonucleoproteins $\mathrm{a} 2 / \mathrm{b} 1$ & 37407 & 0.57486 \\
\hline D2JYH4 & D2JYH4 & actin, aortic smooth muscle & 41982 & 0.56132 \\
\hline B2RDW1 & B2RDW1 & ubiquitin and ribosomal protein $\$ 27$ a precursor & 17953 & 0.54504 \\
\hline Q8TES4 & Q8TES4 & isoform 2 of filamin-a & 279843 & 0.52057 \\
\hline P12814 & ACTN1 & alpha-actinin-1 & 102993 & 0.501 \\
\hline Q53GK6 & Q53GK6 & actin, cytoplasmic 1 & 41710 & 0.4983 \\
\hline B3KUK2 & B3KUK2 & superoxide dismutase $[\mathrm{MN}]$, mitochondrial & 24707 & 0.4851 \\
\hline 075369 & FLNB & isoform 1 of filamin-b & 278021 & 0.48375 \\
\hline Q5TZZ9 & Q5TZZ9 & annexin a1 & 38690 & 0.44331 \\
\hline 043707 & ACTN4 & alpha-actinin-4 & 104788 & 0.4385 \\
\hline Q96HX0 & Q96HX0 & tubulin beta- $2 \mathrm{c}$ chain & 49799 & 0.4044 \\
\hline P14618 & KPYM & isoform $\mathrm{m} 2$ of pyruvate kinase isozymes $\mathrm{m} 1 / \mathrm{m} 2$ & 57900 & 0.3754 \\
\hline Q0QET7 & Q0QET7 & glyceraldehyde-3-phosphate dehydrogenase & 36030 & 0.37292 \\
\hline A4UCS8 & A4UCS8 & isoform alpha-enolase of alpha-enolase & 47139 & 0.3717 \\
\hline Q13509 & TBB3 & tubulin beta- 3 chain & 50400 & 0.33322 \\
\hline P00338 & LDHA & isoform 1 of l-lactate dehydrogenase a chain & 36665 & 0.33172 \\
\hline P11021 & GRP78 & hspa5 protein & 72377 & 0.30588 \\
\hline B7Z4V1 & B7Z4V1 & alpha-actinin-2 & 103788 & 0.2234 \\
\hline P07355 & ANXA2 & isoform 2 of annexin a2 & 40386 & 0.2198 \\
\hline A6NMY6 & AXA2L & putative annexin a2-like protein & 38635 & 0.21773 \\
\hline Q1ZYQ1 & Q1ZYQ1 & isoform 1 of tubulin alpha- $3 \mathrm{c} / \mathrm{d}$ chain & 49928 & 0.1477 \\
\hline B4DK16 & B4DK16 & pre-mrna-processing-splicing factor 8 & 273427 & 0.13834 \\
\hline B4DM32 & B4DM32 & translational activator GCN1 & 292558 & 0.02688 \\
\hline
\end{tabular}

\section{Measurement of total intracellular ROS}

Changes in intracellular ROS levels were detected using the oxidant-sensing fluorescent probe CM$\mathrm{H}_{2}$ DCFDA [11]. This probe was loaded into previously subcultured HUVECs at a final concentration of 10 $\mu \mathrm{mol} / \mathrm{L}$, and cultured at $37^{\circ} \mathrm{C}$ for $30 \mathrm{~min}$ in the dark. After incubation, the culture media were washed twice with PBS (10 mM phosphate buffer, $2.7 \mathrm{mM} \mathrm{KCl}$, and $137 \mathrm{mM} \mathrm{NaCl}, \mathrm{pH} 7.4$ ) to remove the dyes from the media, and analyzed using laser confocal microscopy. Fluorescence images were collected with a laser confocal system mounted onto an inverted microscope equipped with an argon-krypton laser. For studies with $\mathrm{CMH}_{2}$ DCFDA, an excitation wavelength of $488 \mathrm{~nm}$ (argon laser) and emission wavelength of $515 \mathrm{~nm}$ were used.

\section{Statistical analysis}

Results are presented as the means \pm standard deviation (SD). Data were analyzed by one-way analysis of variance (ANOVA) plus Bonferroni's correction (Student-Newman-Keuls). A P value less than 0.05 was considered statistically significant. 


\section{Cellular Physiology $\quad$ Cell Physiol Biochem 2014;33:479-490 and Biochemistry DOI: 10.1159/000358628 2014 S. Karger AG, Bas

Fig. 1. GO analysis of all differentially expressed proteins. (A) Thirty-two proteins are involved in cellular component, 34 in molecular function, and 31 in biological processes. (B) Protein numbers in the molecular function subgroup. (C) Protein numbers in the cellular components subgroup. (D) Protein numbers in the biological processes subgroup.

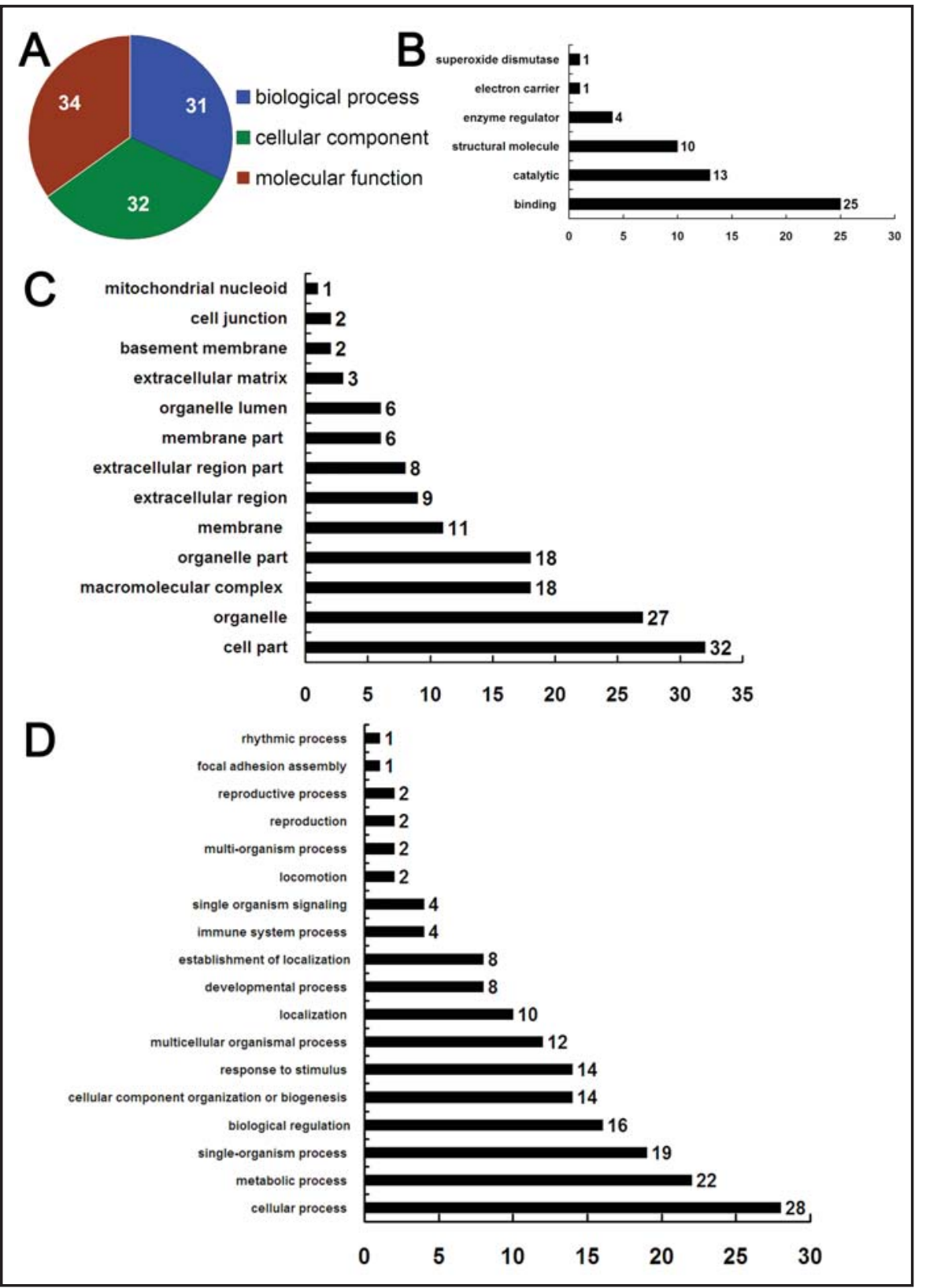

\section{Results}

\section{LC-MS analysis}

There were 39 differentially expressed proteins with an Nratio (the change in the ratio of the up/downregulated proteins induced by uric acid relative to the control) range of 20 $>$ Nratio $>1.5$ (upregulation) or $0.05<$ Nratio $<0.66$ (downregulation). Table 1 shows the differentially expressed proteins.

Gene Ontology analysis of differentially expressed proteins

Differentially expressed proteins were analyzed according to Gene Ontology (GO) classification. Among these proteins, 32 are associated with cellular component, 34 with molecular function, and 31 with biological processes (Fig. 1A). In the molecular function category, 25 proteins are related to binding, while the others are related to catalytic or 
Table 2. 21 proteins are classified to cellular metabolic process

\begin{tabular}{lll}
\hline Uniprot KB & Protein Name & Definition \\
\hline P15121 & ALDR & Aldose reductase, aldo-keto reductase family 1, member B1 \\
Q9UI12 & ATIF1 & ATPase inhibitor, mitochondrial \\
Q00610 & CLH1 & Clathrin heavy chain 1 \\
P11021 & GRP78 & 78 kDa glucose-regulated protein \\
P14618 & KPYM & Pyruvate kinase PKM \\
P00338 & LDHA & L-lactate dehydrogenase A chain \\
P26599 & PTBP1 & Polypyrimidine tract-binding protein 1 \\
P22626 & ROA2 & Heterogeneous nuclear ribonucleoproteins A2/B... \\
P68366 & TBA4A & Tubulin alpha-4A chain \\
Q13509 & TBB3 & Tubulin beta-3 chain \\
B4DTG2 & B4DTG2 & Elongation factor 1-gamma \\
B2RDW1 & B2RDW1 & Ribosomal protein S27a, isoform CRA_c \\
Q1ZYQ1 & Q1ZYQ1 & Tubulin, alpha 2 \\
B3KUK2 & B3KUK2 & Superoxide dismutase \\
Q86SX1 & Q86SX1 & Full-length cDNA 5-PRIME end of clone CS0DN00... \\
Q96HX0 & Q96HX0 & TUBB2C protein \\
Q6IPT9 & Q6IPT9 & Elongation factor 1-alpha \\
B4DTN7 & B4DTN7 & cDNA FLJ57198, highly similar to Protein-glut... \\
B7Z597 & B7Z597 & cDNA FLJ54373, highly similar to 60 kDa heat ... \\
Q9H6X9 & Q9H6X9 & cDNA: FLJ21717 fis, clone COL10322 \\
A4UCS8 & A4UCS8 & Enolase \\
\hline
\end{tabular}

structural molecular function (Fig. 1B). Almost all proteins are related to cell components that determine cell parts, organelles, etc. (Fig. 1C). In the biological process category, 28 proteins are related to cellular processes (Fig. 1D). In this subgroup, 21 proteins are involved in cellular metabolic processes (Table 2). Other biological processes include those of multicellular organisms, e.g., biological regulation and response to stimuli.

\section{Pathway analysis}

Pathway networks involving the differentially expressed proteins were generated using the MAS system (Table 3 and http://yunpan.cn/QpHqtKHsHZMV4). The prominent pathways were those involving EHEC, focal adhesion, gap junctions, leukocyte transendothelial migration, pyruvate metabolism, regulation of actin cytoskeleton, and others. The main proteins involved in these pathways included EZR, TUBA3C, ACTB, TUBB3, TUBB2C, TUBA4A, FLNA, FLNB, ACTN4, ACTN1, among others.

\section{Analyses of ALDR protein}

The ALDR protein, encoded by the aldo-keto reductase family 1 member B1 (AKR1B1) gene, was upregulated $\sim 18$-fold when cells were stimulated with HUA (Table 1). Therefore, we focused on this protein and analyzed the protein-GO networks of all proteins to discover the function of ALDR. The supplementary materials in this paper depict the protein-GO networks of all proteins. The protein-GO networks and pathways related to ALDR indicated that its functions include aldehyde reductase activity, oxidoreductase activity, and electron carrier activity (Fig. 2A), and that it is involved in oxidation reduction, the response to stress or other biological processes, pentose/glucuronate interconversions, and fructose/mannose metabolism or other pathways (Fig. 2B). It is also located in many cellular component (Fig. 2C). The details are listed in Table 4. 


\section{Cellular Physiology $\quad$ Cell Physiol Biochem 2014;33:479-490 \begin{tabular}{l|l|l} 
DOI: 10.1159/000358628 & O 2014 S. Karger AG, Bas
\end{tabular}

Table 3. Kegg Pathway Index

\begin{tabular}{lllll}
\hline Pathway & Count & $\mathrm{p}$-Value & q-Value & Gene \\
\hline Pathogenic Escherichia coli infection - EHEC & 6 & $6.56 \mathrm{E}-13$ & $8.75 \mathrm{E}-13$ & EZR;TUBA3C;ACTB;TUBB3;TUBB2C;TUBA4A \\
Focal adhesion & 5 & $1.27 \mathrm{E}-07$ & $4.63 \mathrm{E}-08$ & FLNA;FLNB;ACTB;ACTN4;ACTN1 \\
Gap junction & 4 & $2.70 \mathrm{E}-07$ & $7.70 \mathrm{E}-08$ & TUBA3C;TUBB3;TUBB2C;TUBA4A \\
Leukocyte transendothelial migration & 4 & $7.76 \mathrm{E}-07$ & $1.94 \mathrm{E}-07$ & EZR;ACTB;ACTN4;ACTN1 \\
Regulation of actin cytoskeleton & 4 & $8.04 \mathrm{E}-06$ & $1.07 \mathrm{E}-06$ & EZR;ACTB;ACTN4;ACTN1 \\
Pyruvate metabolism & 3 & $1.92 \mathrm{E}-06$ & $3.84 \mathrm{E}-07$ & PKM2;AKR1B1;LDHA \\
Glycolysis / Gluconeogenesis & 3 & $7.43 \mathrm{E}-06$ & $1.06 \mathrm{E}-06$ & PKM2;GAPDH;LDHA \\
Adherens junction & 3 & $1.40 \mathrm{E}-05$ & $1.72 \mathrm{E}-06$ & ACTB;ACTN4;ACTN1 \\
Antigen processing and presentation & 3 & $2.00 \mathrm{E}-05$ & $2.00 \mathrm{E}-06$ & HSP90AB1;HSP90AA1;HSPA5 \\
Tight junction & 3 & $7.11 \mathrm{E}-05$ & $5.17 \mathrm{E}-06$ & ACTB;ACTN4;ACTN1 \\
Prostate cancer & 2 & 0.001241361 & $6.37 \mathrm{E}-05$ & HSP90AB1;HSP90AA1 \\
Systemic lupus erythematosus & 2 & 0.003271697 & $1.49 \mathrm{E}-04$ & ACTN4;ACTN1 \\
MAPK signaling pathway & 2 & 0.011417746 & $3.46 \mathrm{E}-04$ & FLNA;FLNB \\
Cysteine metabolism & 1 & 0.007636706 & $2.63 \mathrm{E}-04$ & LDHA \\
Pentose and glucuronate interconversions & 1 & 0.014636105 & $4.30 \mathrm{E}-04$ & AKR1B1 \\
Galactose metabolism & 1 & 0.015217238 & $4.41 \mathrm{E}-04$ & AKR1B1 \\
Propanoate metabolism & 1 & 0.019854431 & $5.59 \mathrm{E}-04$ & LDHA \\
Fructose and mannose metabolism & 1 & 0.021010439 & $5.84 \mathrm{E}-04$ & AKR1B1 \\
Type II diabetes mellitus & 1 & 0.026196251 & $6.99 \mathrm{E}-04$ & PKM2 \\
Glycerolipid metabolism & 1 & 0.027345056 & $7.20 \mathrm{E}-04$ & AKR1B1 \\
Cholera - Infection & 1 & 0.034210528 & $8.66 \mathrm{E}-04$ & ACTB \\
Insulin signaling pathway & 1 & 0.078257149 & 0.001537602 & PKM2 \\
Purine metabolism & 1 & 0.085858363 & 0.001619955 & PKM2 \\
Alzheimer's disease & 1 & 0.099282077 & 0.001772894 & GAPDH \\
\hline & & & & \\
\hline & & & & \\
& 1 & & &
\end{tabular}

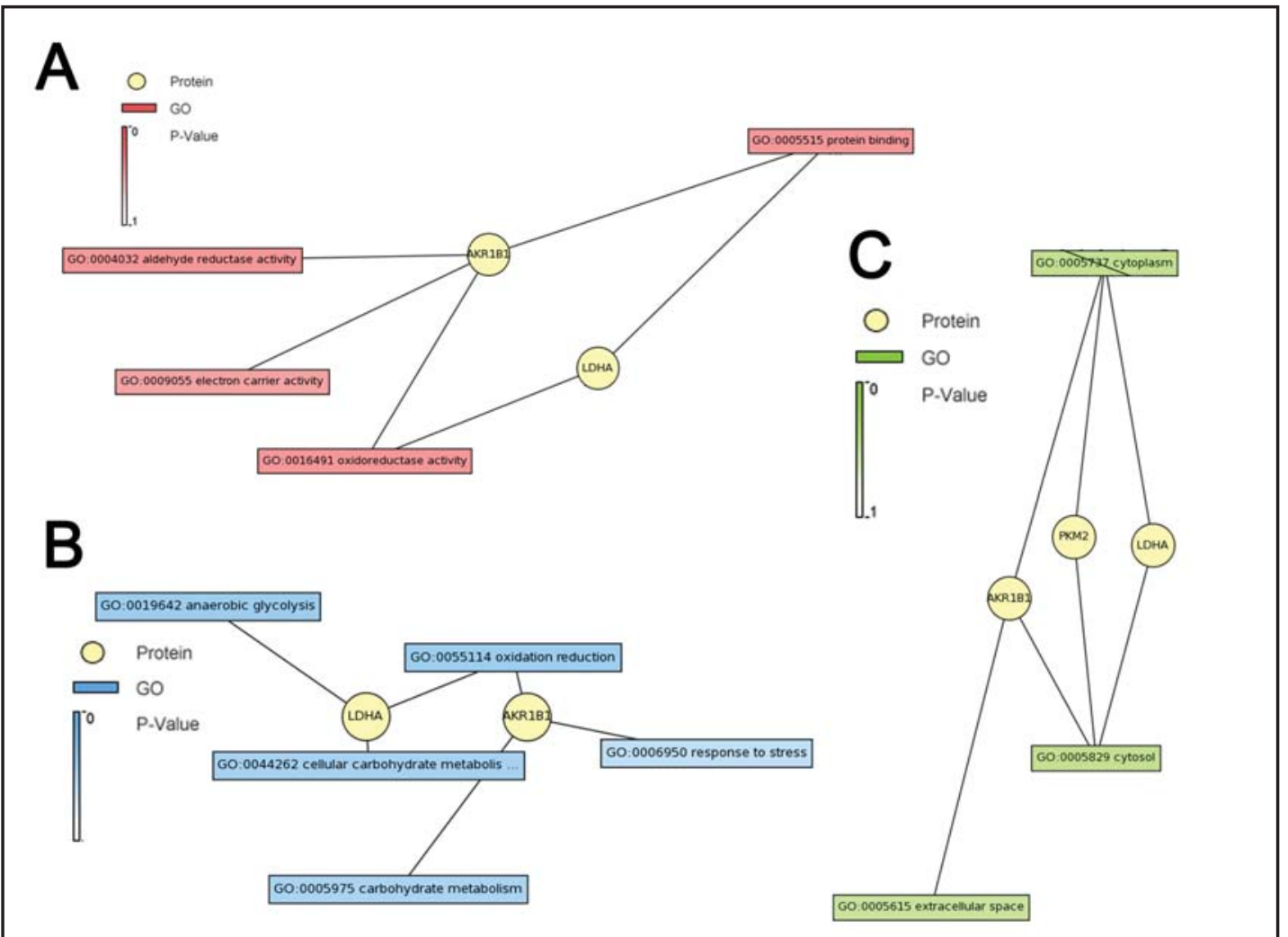

Fig. 2. Protein-GO networks and interactions with the ALDR (AKR1B1) protein. (A) Significant molecular function network of the ALDR protein. (B) Significant cellular structure network of the ALDR protein. (C) Significant biological process network of the ALDR protein. 
Talbe 4. GO and pathway analysis of ALDR

\begin{tabular}{llll}
\hline Molecular Function & Biological Process & Cellular Component & Pathway \\
\hline $\begin{array}{l}\text { G0:0004032 aldehyde } \\
\text { reductase activity }\end{array}$ & G0:0005975 carbohydrate & GO:0005615 & $\begin{array}{l}\text { Pentose and glucuronate } \\
\text { interconversions }\end{array}$ \\
G0:0005515 protein binding & GO:0006950 response to & GO:0005737 & Fructose and mannose \\
G0:0009055 electron carrier & stress & cytoplasm & metabolism \\
activity & GO:0055114 oxidation & GO:0005829 cytosol & Galactose metabolism \\
G0:0016491 oxidoreductase & reduction & & Glycerolipid metabolism \\
activity & & & Pyruvate metabolism \\
\hline
\end{tabular}

Talbe 5. Diseases related with ALDR. OMIM: Online Mendelian Inheritance in Man (OMIM database) ; GAD: The Genetic Association Database

\begin{tabular}{ll}
\hline Name & Source \\
\hline OMIM 103880 & OMIM \\
OMIM 103188 & OMIM \\
nephropathy in other diseases & GAD \\
hypospadias & GAD \\
microangiopathy & GAD \\
neuropathy, diabetic & GAD \\
microvascular complications & GAD \\
diabetes, type 1 & GAD \\
diabetic glomerulopathy; glomerulopathy, diabetic; kidney failure; & GAD \\
retinopathy, diabetic & GAD \\
nephropathy, diabetic; retinopathy, diabetic & GAD \\
diabetes, type 2 & GAD \\
Type 2 diabetes & GAD \\
\hline
\end{tabular}

Combining these results, we analyzed the interacting proteins and diseases related to ALDR using MAS. The ALDR protein interacted with many other proteins (see http:// yunpan.cn/QpHqtKHsHZMV4), including IKBKE, ZNF253, CDC, and others. Diseases related to ALDR are listed in Table 5, including nephropathy, diabetes, microvascular complications, and retinopathies. This suggests that the ALDR protein plays an important role in the vascular disease process.

\section{ALDR in a hyperuricemia mouse model and in HUVECS}

Sera derived from hyperuricemic mice and normal control mice were used to detect levels of UA, while aortas were used for immunohistochemistry. The results indicate that serum UA levels were clearly higher in the hyperuricemic mice than in the control group (Fig. 3A). Expression of ALDR was upregulated in the aortas of the hyperuricemic mice compared with the normal controls (Fig. 3B). The cell extract from HUVECs was subjected to Western blotting to determine ALDR protein expression, which was significantly enhanced in HUVECs cultured with HUA (Fig. 3C).

\section{ROS production in endothelial cells decreased after ALDR inhibition}

It was confirmed that HUA can lead to ALDR protein overexpression in HUVECs in vitro. HUVECs were transfected with siALDR to inhibit ALDR. To examine ALDR protein expression, the extract was examined using Western blot analysis. Overexpression of ALDR was inhibited (Fig. 4A), indicating that siALDR effectively inhibited ALDR protein expression.

Our recent research has demonstrated that HUA induces endothelial injury via ROS production. We used siALDR to downregulate the ALDR protein and subsequently measured ROS levels. The results show that intracellular ROS levels were lower in HUVECs transfected with siALDR and incubated with HUA than in non-siRNA transfection cells (Fig. 4B). 


\section{Cellular Physiology Cell Physiol Biochem 2014;33:479-490 \begin{tabular}{l|l} 
DOI: 10.1159/000358628 & O 2014 S. Karger AG, Basel
\end{tabular} and Biochemistry Published online: February 14, 2014 www.karger.com/cpb

Fig. 3. ALDR protein expression in vivo and in vitro. (A) Hyperuricemic mice were injected intraperitoneally with oxonic acid potassium salt and UA for 14 days; serum UA levels increased significantly $\left(^{*} p<0.05\right.$ compared with controls, $n=6)$. (B) ALDR protein expression was enhanced in mouse aortas, as determined by immunohistochemistry ( $\times 200)$. (C) ALDR protein expression increased in HUVECs cultured with $600 \mu \mathrm{M}$ UA for $24 \mathrm{~h}\left({ }^{*} p<0.05 v s\right.$. control, $\left.\mathrm{n}=6\right)$.

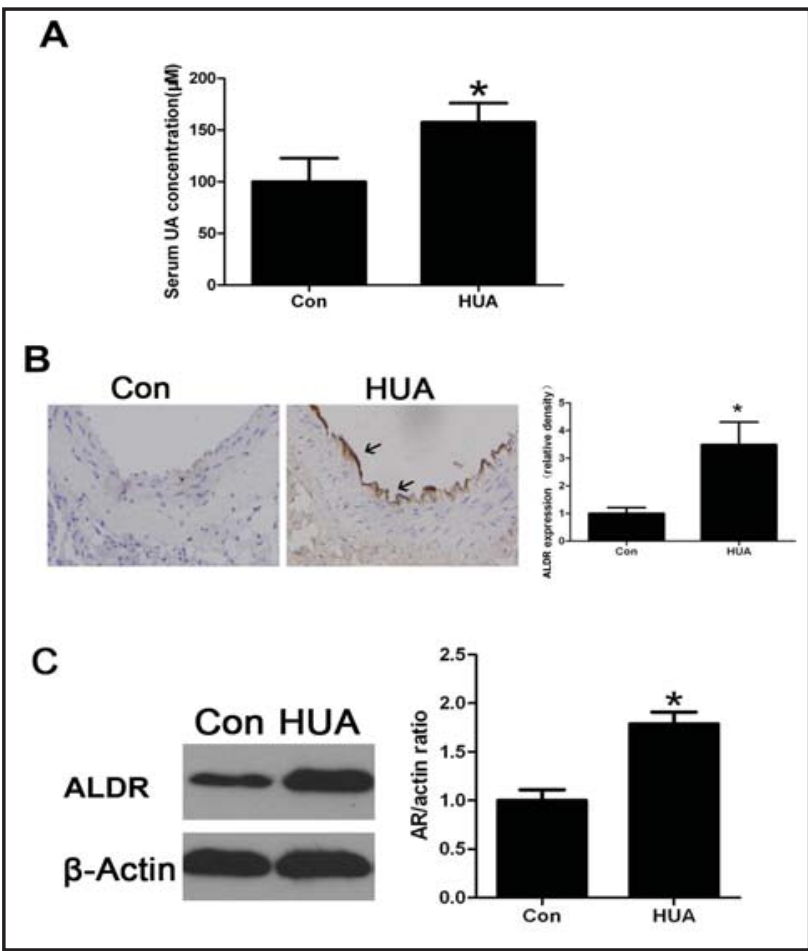

Fig. 4. ALDR protein expression and ROS levels in HUVECs after transfection with siRNA. (A) ALDR protein expression was decreased in HUVECs cultured in medium containing $600 \mu \mathrm{M}$ UA after transfection with siALDR to inhibit the ALDR protein $\left({ }^{*} p<0.05 v s\right.$. siCon group, $n=6$ ). (B) HUVECs were transfected with siALDR and treated with HUA for $24 \mathrm{~h}$; ROS production decreased compared with the non-siRNA transfection group $\left({ }^{*} p<0.05\right.$ vs. siCon group, $\mathrm{n}=6$ ).

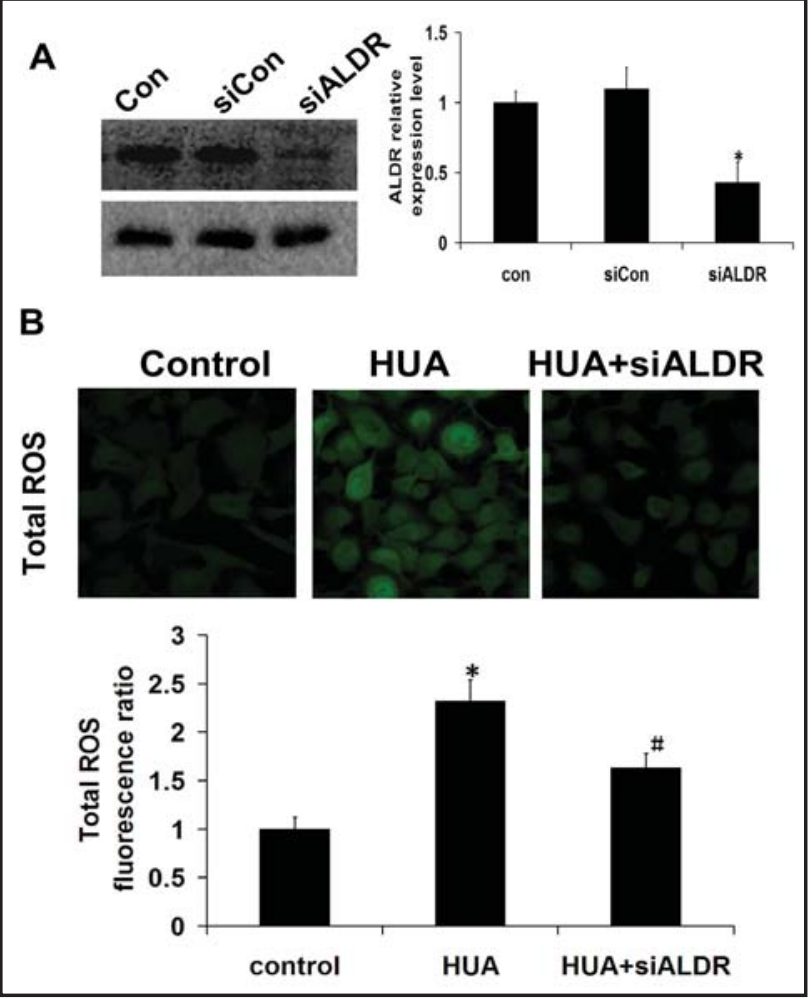

\section{Discussion}

The number of hyperuricemia cases is increasing worldwide. Hyperuricemia has been confirmed as an independent risk factor for CKD and cardiovascular disease, among other ailments $[12,13]$. Hyperuricemia induces endothelial injury, but the mechanism has not 


\section{Cellular Physiology and Biochemistry}

Cell Physiol Biochem 2014;33:479-490

\begin{tabular}{l|l}
\hline DOI: $10.1159 / 000358628$ & (c) 2014 S. Karger AG, Basel
\end{tabular}

Published onlıne: February 14, 2014

www.karger.com/cpb

been well investigated [14]. Several reports have analyzed differentially expressed proteins in the development of hyperuricemia. Our objective was to identify the differentially expressed proteins in HUVECs cultured with HUA. Therefore, we analyzed differentially expressed protein profiles using SILAC and LC-MS/MS. Proteomics applied on a large scale may provide useful information. The results identified 39 differentially expressed proteins among three GO groups, 32 of which contributed to cellular component, 34 to molecular function, and 31 to biological processes, particularly ALDR protein upregulation. ALDR is an aldehyde-metabolizing enzyme, a member of the aldo-keto reductase superfamily, and a regulator of ROS signaling [15]. Previous studies have confirmed that the ALDR protein plays a key role in various diseases, such as diabetic nephropathy and gastrointestinal neoplasia [15-17]. Based on the above results, we analyzed the protein-GO networks of all proteins and found that ALDR possesses aldehyde reductase, oxidoreductase, electron carrier, and other activities. Changes in ALDR are related to endothelial cell injury in uremia [18], and this function is associated with ROS, which can be generated by several mechanisms [13, 19]. Activation of the polyol pathway has been observed in diabetes and can lead to diabetic microvascular dysfunction via oxidative stress [20]. Expression of ALDR protein and mRNA is upregulated in diabetic rats compared with normal controls; superoxide anions and malondialdehyde (MDA) were increased concomitantly, while superoxide dismutase (SOD) was decreased in cultured mesangial cells treated with high glucose; inhibition of ALDR and reduced oxidative stress ameliorated renal injury $[21,22]$. We also detected upregulation of the ALDR protein in other diseases, including hyperlipidemia and atherosclerosis [23]. Researchers have confirmed that transforming growth factor- $\beta 1$ (TGF- $\beta 1$ ) induces upregulation of ALDR through activation of ROS and the nuclear factor-erythroid 2-related factor 2 (Nrf2) pathway in human renal mesangial cells (HRMCs) [24]. High glucose leads to ALDR overexpression by increasing NF- $\mathrm{KB}$ binding activity, and silencing the NF- $\kappa B$ genes can reverse ALDR overexpression [25]. Some studies have also found that the expression of ALDR is mediated by TonEBP to maintain hypertonicity in the kidney medulla [26, 27]. These proteins were not found in the 39 differentially expressed proteins in our experiment and were not significantly differentially expressed in the HUA group compared with the control group (data not shown), but this result may be limited by the precision and sensitivity of the detection method. No research has examined the relationship between HUA and ALDR, and this is the first report that ALDR levels are upregulated in HUVECs cultured with HUA. The mechanism by which HUA induces overexpression of ALDR is unknown, but we hypothesize that HUA increases ALDR by altering normal physiological functions. Understanding the exact mechanism by which HUA induces ALDR overexpression will require further studies. NADPH is a co-factor for the ALDR protein and a substrate in the generation of GSH; the ALDR protein depletes NADPH and consequently decreases GSH, such that the antioxidant capacity is impaired by the increased levels of ALDR protein $[20,28]$. Based on these results, we propose a relationship between ALDR and ROS. Also, intracellular levels of ROS blocked by siALDR were lower than those of the non-siRNA transfection group. We speculate that the ALDR protein plays an important role in endothelial injury caused by hyperuricemia via oxidative stress. However, the precise mechanism remains unclear; thus, further studies are needed to explain the relationship between ALDR and endothelial injury.

\section{Conclusion}

Our work provides an overview of the proteomic variations in HUVECs treated with HUA. In this study, SILAC combined with LC-MS/MS analysis was used to determine the differentially expressed protein profiles in HUVECs treated with HUA. We found that expression of the ALDR protein increased significantly and that ROS levels were related to ALDR protein levels. This study also revealed that ALDR plays a vital role in endothelial injury and oxidative stress induced by HUA. Thus, our results may facilitate a more complete understanding of the mechanism behind endothelial injury induced by HUA. 


\section{Cellular Physiology \\ and Biochemistry}

Cell Physiol Biochem 2014;33:479-490

\begin{tabular}{l|l}
\hline DOI: $10.1159 / 000358628$ & (c) 2014 S. Karger AG, Basel
\end{tabular}

www.karger.com/cpb

Zhang et al.: ALDR Enhanced ECs Injury in Hyperuricemia

\section{Acknowledgements}

This work is supported by Chinese National Natural Sciences Foundation (No. 81102673 and No. 31170810) and Beijing NOVA Program (Z121107002512078).

\section{References}

1 Wu XW, Muzny DM, Lee CC, Caskey CT: Two independent mutational events in the loss of urate oxidase during hominoid evolution. J Mol Evol 1992;34:78-84.

2 Alvarez-Lario B, Macarron-Vicente J: Uric acid and evolution. Rheumatology (Oxford) 2010;49:2010-2015.

-3 Sautin YY, Johnson RJ: Uric acid: the oxidant-antioxidant paradox. Nucleosides Nucleotides Nucleic Acids 2008;27:608-619.

4 Kanbay M, Segal M, Afsar B, Kang DH, Rodriguez-Iturbe B, Johnson RJ: The role of uric acid in the pathogenesis of human cardiovascular disease. Heart 2013;99:759-766.

-5 Wang Y, Bao X: Effects of uric acid on endothelial dysfunction in early chronic kidney disease and its mechanisms. Eur J Med Res 2013;18:26.

6 Park JH, Jin YM, Hwang S, Cho DH, Kang DH, Jo I: Uric acid attenuates nitric oxide production by decreasing the interaction between endothelial nitric oxide synthase and calmodulin in human umbilical vein endothelial cells: a mechanism for uric acid-induced cardiovascular disease development. Nitric Oxide 2013;32:36-42.

7 Sanchez-Lozada LG, Lanaspa MA, Cristobal-Garcia M, Garcia-Arroyo F, Soto V, Cruz-Robles D, Nakagawa T, Yu MA, Kang DH, Johnson RJ: Uric Acid-induced endothelial dysfunction is associated with mitochondrial alterations and decreased intracellular ATP concentrations. Nephron Exp Nephrol 2012;121:e71-78.

-8 Yu MA, Sanchez-Lozada LG, Johnson RJ, Kang DH: Oxidative stress with an activation of the reninangiotensin system in human vascular endothelial cells as a novel mechanism of uric acid-induced endothelial dysfunction. J Hypertens 2010;28:1234-1242.

-9 Quan H, Peng X, Liu S, Bo F, Yang L, Huang Z, Li H, Chen X, Di W: Differentially expressed protein profile of renal tubule cell stimulated by elevated uric acid using SILAC coupled to LC-MS. Cell Physiol Biochem 2011; 27:91-98.

10 Yang Z, Xiaohua W, Lei J, Ruoyun T, Mingxia X, Weichun H, Li F, Ping W, Junwei Y: Uric acid increases fibronectin synthesis through upregulation of lysyl oxidase expression in rat renal tubular epithelial cells. Am J Physiol Renal Physiol 2010;299:F336-346.

-11 Turturro F, Friday E, Welbourne T: Hyperglycemia regulates thioredoxin-ROS activity through induction of thioredoxin-interacting protein (TXNIP) in metastatic breast cancer-derived cells MDA-MB-231. BMC Cancer 2007; 7:96.

$\checkmark 12$ Filiopoulos V, Hadjiyannakos D, Vlassopoulos D: New insights into uric acid effects on the progression and prognosis of chronic kidney disease. Ren Fail 2012;34:510-520.

-13 Puddu P, Puddu GM, Cravero E, Vizioli L, Muscari A: Relationships among hyperuricemia, endothelial dysfunction and cardiovascular disease: molecular mechanisms and clinical implications. J Cardiol 2012;59:235-242.

14 Johnson RJ, Kang DH, Feig D, Kivlighn S, Kanellis J, Watanabe S, Tuttle KR, Rodriguez-Iturbe B, HerreraAcosta J, Mazzali M: Is there a pathogenetic role for uric acid in hypertension and cardiovascular and renal disease? Hypertension 2003;41:1183-1190.

15 Tammali R, Reddy AB, Ramana KV, Petrash JM, Srivastava SK: Aldose reductase deficiency in mice prevents azoxymethane-induced colonic preneoplastic aberrant crypt foci formation. Carcinogenesis 2009;30:799807.

16 Oates PJ: Aldose reductase, still a compelling target for diabetic neuropathy. Curr Drug Targets 2008;9:1436.

17 Srivastava SK, Ramana KV, Bhatnagar A: Role of aldose reductase and oxidative damage in diabetes and the consequent potential for therapeutic options. Endocr Rev 2005;26:380-392. 


\section{Cellular Physiology and Biochemistry}

Cell Physiol Biochem 2014;33:479-490

\begin{tabular}{l|l}
\hline DOI: $10.1159 / 000358628$ & (c) 2014 S. Karger AG, Basel
\end{tabular}

18 Carbo C, Arderiu G, Escolar G, Fuste B, Cases A, Carrascal M, Abian J, Diaz-Ricart M: Differential expression of proteins from cultured endothelial cells exposed to uremic versus normal serum. Am J Kidney Dis 2008;51:603-612.

19 Zhang DM, Li YC, Xu D, Ding XQ, Kong LD: Protection of curcumin against fructose-induced hyperuricaemia and renal endothelial dysfunction involves NO-mediated JAK-STAT signalling in rats. Food Chem 2012;134:2184-2193.

20 Nakamura N, Obayashi H, Fujii M, Fukui M, Yoshimori K, Ogata M, Hasegawa G, Shigeta H, Kitagawa Y, Yoshikawa T, et al: Induction of aldose reductase in cultured human microvascular endothelial cells by advanced glycation end products. Free Radic Biol Med 2000;29:17-25.

-21 Liu W, Liu P, Tao S, Deng Y, Li X, Lan T, Zhang X, Guo F, Huang W, Chen F, et al: Berberine inhibits aldose reductase and oxidative stress in rat mesangial cells cultured under high glucose. Arch Biochem Biophys 2008;475:128-134.

-22 Liu WH, Hei ZQ, Nie H, Tang FT, Huang HQ Li XJ, Deng YH, Chen SR, Guo FF, Huang WG, et al: Berberine ameliorates renal injury in streptozotocin-induced diabetic rats by suppression of both oxidative stress and aldose reductase. Chin Med J (Engl) 2008;121:706-712.

23 Gleissner CA, Sanders JM, Nadler J, Ley K: Upregulation of aldose reductase during foam cell formation as possible link among diabetes, hyperlipidemia, and atherosclerosis. Arterioscler Thromb Vasc Biol 2008;28:1137-1143.

24 Wang F, Tian F, Whitman SA, Zhang DD, Nishinaka T, Zhang N, Jiang T: Regulation of transforming growth factor beta1-dependent aldose reductase expression by the Nrf2 signal pathway in human mesangial cells. Eur J Cell Biol 2012;91:774-781.

25 Yang B, Hodgkinson A, Oates PJ, Millward BA, Demaine AG: High glucose induction of DNA-binding activity of the transcription factor NFkappaB in patients with diabetic nephropathy. Biochim Biophys Acta 2008;1782:295-302.

26 Miyakawa H, Woo SK, Dahl SC, Handler JS, Kwon HM: Tonicity-responsive enhancer binding protein, a rel-like protein that stimulates transcription in response to hypertonicity. Proc Natl Acad Sci USA 1999;96:2538-2542.

27 Neuhofer W, Fraek ML, Beck FX: Nitric oxide decreases expression of osmoprotective genes via direct inhibition of TonEBP transcriptional activity. Pflugers Arch 2009;457:831-843.

28 Chung SS, Ho EC, Lam KS, Chung SK: Contribution of polyol pathway to diabetes-induced oxidative stress. J Am Soc Nephrol 2003;14:S233-236. 